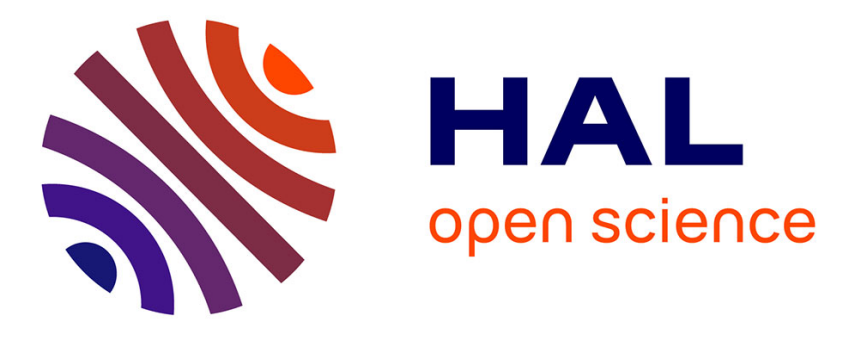

\title{
Low-noise dual-frequency laser for compact Cs atomic clocks
}

\author{
Paul Dumont, Fabiola Camargo, Jean-Marie Danet, David Holleville, \\ Stéphane Guerandel, Grégoire Pillet, Ghaya Baili, Loïc Morvan, Daniel Dolfi, \\ Iryna Gozhyk, et al.
}

\section{To cite this version:}

Paul Dumont, Fabiola Camargo, Jean-Marie Danet, David Holleville, Stéphane Guerandel, et al.. Low-noise dual-frequency laser for compact Cs atomic clocks. Journal of Lightwave Technology, 2014, 32 (20), pp.3817-3823. 10.1109/JLT.2014.2318179 . hal-01060557

\section{HAL Id: hal-01060557 \\ https://hal-iogs.archives-ouvertes.fr/hal-01060557}

Submitted on 3 Sep 2014

HAL is a multi-disciplinary open access archive for the deposit and dissemination of scientific research documents, whether they are published or not. The documents may come from teaching and research institutions in France or abroad, or from public or private research centers.
L'archive ouverte pluridisciplinaire HAL, est destinée au dépôt et à la diffusion de documents scientifiques de niveau recherche, publiés ou non, émanant des établissements d'enseignement et de recherche français ou étrangers, des laboratoires publics ou privés. 


\title{
Low-noise dual-frequency laser for compact Cs atomic clocks
}

\author{
Paul Dumont, Fabiola Camargo, Jean-Marie Danet, David Holleville, Stéphane Guerandel, Grégoire \\ Pillet, Ghaya Baili, Loïc Morvan, Daniel Dolfi, Iryna Gozhyk, Grégoire Beaudoin, Isabelle Sagnes, \\ Patrick Georges, Gaëlle Lucas-Leclin
}

\begin{abstract}
We report the dual-frequency and dual-polarization emission of an optically-pumped vertical external-cavity semiconductor laser (OP-VECSEL). Our laser source provides a high-purity optically-carried RF signal tunable in the GHz range, and is specifically designed for the coherent population trapping (CPT) of Cs atoms in compact atomic clocks. The laser spectrum is stabilized onto a Cs atomic transition at $852.1 \mathrm{~nm}$, and the frequency difference is locked to a local oscillator at $9.2 \mathrm{GHz}$. Special attention has been paid to the evaluation of the frequency, intensity and phase noise properties. A maximum phase noise of $90 \mathrm{dBrad}^{2} / \mathrm{Hz}$ has been measured. Finally, we estimate the contribution of the laser noise on the short-term frequency stability of a CPT atomic clock, and predict that a value below $3 x$ $10^{-13}$ over one second is a realistic target.
\end{abstract}

Index Terms-Microwave photonics, Vertical cavity surface emitting lasers, Clocks, Laser noise.

\section{INTRODUCTION}

A TOMIC frequency references provide high-precision stable signals, which are crucial in the most demanding applications as satellite positioning, high bitrate communication networks, or high-end inertial navigation. Because of their potential for size reduction, atomic clocks based on the coherent population trapping (CPT) of ${ }^{133} \mathrm{Cs}$ atoms have raised a considerable interest within the last decade in the scientific and industrial community [1], [2]. In a CPT-based atomic clock, the microwave interrogation that delivers the atomic reference signal is induced by two phase-coherent laser fields at resonance with a common excited state. When the frequency difference between the two laser fields corresponds exactly to the ground-state hyperfine splitting (equal to 9.192 $631770 \mathrm{GHz}$ with ${ }^{133} \mathrm{Cs}$ atoms), the atoms are trapped in a dark state and stop interacting with the laser light. The resulting narrow transmission line in the atomic absorption spectrum is used as a frequency reference. In order to guarantee the stability

Manuscript received January $15^{\text {th }} 2014$

Part of this work was funded by the Agence Nationale de la Recherche [ANR-07-BLAN-0320-03] and by the French RENATECH network. The authors gratefully aknowledge support by Triangle de la Physique [2010-089T]; the Direction Générale de l'Armement; the Labex First-TF.

Fabiola Camargo, Gaelle Lucas-Leclin and Patrick Georges are with the Laboratoire Charles Fabry, Institut d'Optique, CNRS, Univ Paris-Sud 11, Palaiseau, 91127, France (email: gaelle.lucas-leclin@institutoptique.fr).

Jean-Marie Danet, David Holleville and Stéphane Guérandel are with the LNE-SYRTE, Systèmes de Référence Temps-Espace, Observatoire de Paris, CNRS, UPMC, Paris, 75014, France (email: stephane.guerandel@obspm.fr). of the clock frequency, CPT atomic clocks require a high purity, optically-carried, microwave signal as well as low-noise properties for the laser source (intensity and frequency fluctuations). Among the different optical configurations which influence the CPT signal, those combining cross-polarized excitation scheme and temporal Ramsey-like pulsed interrogation have demonstrated highly contrasted and narrowlinewidth CPT resonances [3]. Such a configuration was recently proven to reach a short-term frequency stability of 3.2 $\times 10^{-13} \tau^{-1 / 2}$ [4]. However those high performance CPT clocks use bulky laser architectures (with two lasers [3] or one laser, a modulator and a Michelson interferometer [5]) in order to generate both coherent and cross-polarized laser modes. Such solutions have proven to fit in compact housing, but the reduction of their volume, while keeping their clock frequency stability in the order of $10^{-13}$ over one second, remains a main challenge.

We propose here a new laser source for compact CPT clocks which improves the trade-off performance vs size. It consists in a unique dual-frequency and dual polarization laser. Indeed, the dual-frequency emission of a solid-state laser has been demonstrated in the $\mathrm{GHz}$ range with a Er:Yb:Glass laser [6] and in the $\mathrm{THz}$ range with a $\mathrm{Yb}: \mathrm{KGW}$ [7], both based on the simultaneous emission of two cross-polarized longitudinal modes inside the same laser cavity. However, the main limitation of these lasers is their strong intensity noise inherent to their class-B dynamical behavior. In order to overcome this limitation, a dual-frequency emission has been recently demonstrated in a class A optically pumped vertical-externalcavity semiconductor laser (OP-VECSEL) which does not suffer from relaxation oscillation since the photon lifetime inside the cavity is much longer than the excited carriers lifetime [8]. This configuration has been demonstrated at $1 \mu \mathrm{m}$ [9] and $852 \mathrm{~nm}[10]$.

Grégoire Pillet, Ghaya Baili, Loïc Morvan and Daniel Dolfi are with Thales Research \& Technology - France, Palaiseau, 91767, France (gregoire.pillet@thalesgroup.com).

Grégoire Beaudoin and Isabelle Sagnes are with Laboratoire de Photonique et de Nanostructures, CNRS UPR20, Marcoussis, 91460, France (email: isabelle.sagnes@lpn.cnrs.fr).

Paul Dumont is with the Laboratoire Charles Fabry, Institut d'Optique, CNRS, Univ Paris-Sud 11 and also with the LNE-SYRTE, Systèmes de Référence Temps-Espace, Observatoire de Paris, CNRS, UPMC.

Iryna Gozhyk is with Laboratoire de Photonique et de Nanostructures, CNRS UPR20 and also with Laboratoire Charles Fabry, Institut d'Optique, CNRS, Univ Paris-Sud 11. 
We investigate the dual-frequency and dual polarization OPVECSEL emitting at the Cs $D_{2}$ line $(\lambda=852 \mathrm{~nm})$ with a frequency difference tunable around the Cs ground state splitting, and noise properties compatible with the coherent population trapping of Cs atoms.

This paper is organized as follows: the design of the dualfrequency OP-VECSEL is described in section II; the tunability of the frequency difference between the two modes is also detailed. Section III presents the laser characterization under free-running operation and section IV describes the stabilization set-up of the laser emission. Finally section V is dedicated to the measurements of the noise properties of the dual-frequency laser, which are used in section VI to estimate the laser noise contribution to the clock frequency stability.

\section{DESIGN OF THE DUAL-FREQUENCY AND DUAL- POLARIZATION OP-VECSEL}

The optically-pumped VECSEL design enables the introduction of free-space intracavity elements to force the dual-frequency and dual-polarization emission of the cavity, while using specially designed semiconductor structure emitting at the targeted wavelength [11].

\section{A. Semiconductor structure}

The semiconductor chip is a conventional multilayer active structure, grown with metal-organic chemical-vapor deposition on a $350 \mu \mathrm{m}$-thick GaAs substrate. It is carefully designed to provide light emission at $852 \mathrm{~nm}$ and to tolerate optical losses of a few percents that are introduced by the intra-cavity elements (cf II.B). We take advantage of our previous works on the design of $\mathrm{AlGaAs} / \mathrm{GaAs}$ semiconductor active chips at 852 $\mathrm{nm}$, which have demonstrated up to $300 \mathrm{~mW}$ at the $\mathrm{Cs}_{2} \mathrm{D}_{2}$ line [12].

A distributed Bragg reflector (DBR), composed of 32.5 pairs of $\lambda / 4$-thick AlAs $/ \mathrm{Al}_{0.225} \mathrm{Ga}_{0.775}$ As layers, provides a $80-\mathrm{nm}$ large high reflectivity centered at $850 \mathrm{~nm}$, with $R>99.94 \%$ at $852 \mathrm{~nm}$. The $30 \lambda / 4$-thick active structure is composed of seven $8 \mathrm{~nm}$-thick GaAs quantum wells (QW) embedded in pumpabsorbing $\mathrm{Al}_{0.225} \mathrm{Ga}_{0.775} \mathrm{As}$ barriers, absorbing up to $90 \%$ of the internal pump power in a single pass. The QWs are placed at the antinodes of the optical standing wave created inside the active chip. Two 30-nm thick $\mathrm{Al}{ }_{0.39} \mathrm{Ga}_{0.61} \mathrm{As}$ layers form potential barriers on each side of the active cavity, for carrier confinement. Finally, a $50 \mathrm{~nm}$-thick $\mathrm{InGaP}$ capping layer protects the cavity from Al-oxidation. The design of this structure, without anti-reflection coating, results in an enhancement of the modal gain thanks to constructive interferences inside the semiconductor sub-cavity.

\section{B. Laser cavity}

The laser cavity is composed of the semiconductor chip and a $R=15 \mathrm{~mm}$ concave output coupler, transmitting $0.5 \%$ at 850 $\mathrm{nm}$. The pump source is a broad-area laser diode emitting at 670 $\mathrm{nm}$, coupled into a $100 \mu \mathrm{m}$ diameter, $N A=0.22$, multimode fiber. This source delivers up to $1 \mathrm{~W}$ at the fiber end. The pump beam is focused on the semiconductor chip with two doublets $\left(f_{1}=25 \mathrm{~mm}, f_{2}=19 \mathrm{~mm}\right)$ under a $50^{\circ}$ angle, yielding a $70 \mu \mathrm{m}$

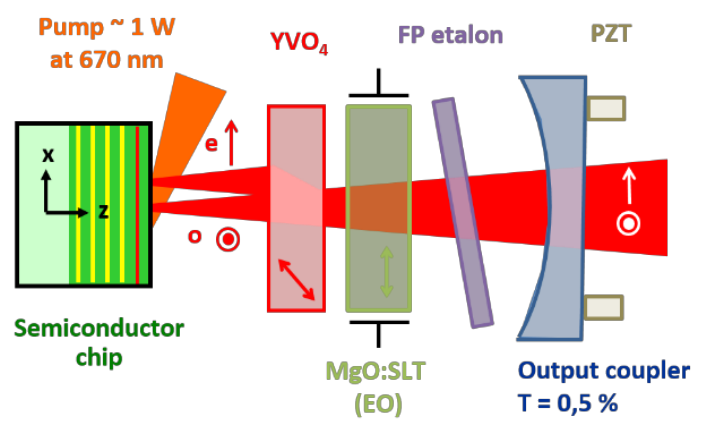

Fig. 1. Dual frequency laser cavity including the semiconductor chip and all three intracavity elements. FP etalon: Fabry-Pérot etalon; PZT: piezotransducer, EO: Electro-optic.

by $110 \mu \mathrm{m}$-elliptical spot on the semiconductor structure.

The dual-polarization emission is obtained by introducing a birefringent element in the laser cavity, which raises the degeneracy between polarizations and thus results in the stable oscillation of two cross-polarized modes (Fig. 1). Here we use a $500 \mu \mathrm{m}$-thick antireflection coated $\mathrm{YVO}_{4}$ plate cut at $45^{\circ}$ to its optical axis, which induces a spatial separation $d$ of $50 \mu \mathrm{m}$ between the extraordinary and ordinary beams in the structure along the longer axis of the pump ellipse. This spatial separation reduces the coupling between the two polarizations which ensures the stable dual-polarization, dual-frequency emission [13]; nevertheless the spatial separation is small enough to maintain a good overlap between the pump beam and the two laser spots. Finally, the single-frequency operation at each polarization, as well as the wavelength coarse tunability, are obtained using a $50 \mu \mathrm{m}$-thick uncoated silica etalon with a free spectral range $F S R_{\text {Etalon }}=2 \mathrm{THz}$. Fine tuning of the laser wavelength is obtained using a piezo-transducer glued on the output coupler. To control the laser frequency difference, a $5 \%$ $\mathrm{MgO}-$ doped stoichiometric lithium tantalate (SLT) crystal is placed within the cavity. Its high transparency at $852 \mathrm{~nm}$ minimizes additional intra-cavity losses. The crystal axes are oriented to limit the ordinary-polarized mode frequency dependency with the SLT applied voltage. Both thermo-optic (TO) and electro-optic (EO) properties of the MgO:SLT enable a broad tunability of the cavity birefringence. With all intracavity elements, the FSR of the laser cavity is $F_{\text {cav }}=12$ $\mathrm{GHz}$, higher than the targeted frequency difference $\Delta v_{0}=$ $9.192 \mathrm{GHz}$, and corresponding to an optical cavity length $L_{c a v}$ $=12.5 \mathrm{~mm}$.

The laser cavity design has focused on compactness as well as high mechanical and thermal stability. The pump optics, the semiconductor chip and the intra-cavity elements are integrated in a compact $90 \mathrm{~mm} \times 90 \mathrm{~mm} \times 40 \mathrm{~mm}$ casing. This limits mechanical and acoustic vibrations as well as air temperature fluctuations inside the external cavity. The temperature of the whole set-up is stabilized to $24^{\circ} \mathrm{C}$. The semiconductor chip temperature is maintained at $16^{\circ} \mathrm{C}$ using a Peltier element.

\section{Frequency difference tunability}

The birefringence introduced inside the cavity generates two combs of cross-polarized longitudinal modes. For two adjacent cross-polarized oscillating modes (i.e. with a frequency 
difference below $F S R_{c a v}$ ), the laser beatnote frequency, defined as the frequency difference $\Delta v=\left|v_{\text {ext }}-v_{\text {ord }}\right|$, is given by the formula:

$$
\Delta v=F S R_{c a v}\left|\frac{2 \Delta L}{\lambda}\right|
$$

where $\Delta L=L_{c a v}^{e x t}-L_{c a v}^{\text {ord }}$ corresponds to the optical path difference due to the birefringence inside the cavity, and is defined modulo $\lambda / 2$. Thus $\Delta v$ is tunable over one FSR of the cavity. The tunability of $\Delta L$ is calculated from the contributions of both $\mathrm{YVO}_{4}$ and SLT crystals, taking into account three main effects: thermal expansion, thermo-optic and electro-optic effects. The birefringence variation of SLT with the applied transverse voltage follows the relationship:

$$
\Delta n_{S L T}^{E O}(V)=\frac{V}{e}\left(\frac{n_{e}^{3}}{2} r_{33}-\frac{n_{o}^{3}}{2} r_{13}\right)
$$

where $e=2 \mathrm{~mm}$ is the distance between the two electrodes used to apply a voltage $V$ on the crystal, $n_{e}$ and $n_{o}$ are the refraction indexes for the extraordinary and ordinary polarizations, $r_{33}$ and $r_{13}$ are the electro-optic coefficients along the axes $\mathrm{x}$ and $\mathrm{z}$, respectively ( $\mathrm{x}$ is the axis on which the voltage is applied and $\mathrm{z}$ is along the beam propagation). The contribution of thermal changes on birefringence can be estimated with the following relationship:

$$
\frac{\partial(\Delta L)}{\partial T}=\frac{\partial L}{\partial T}\left(n_{e}-n_{o}\right)+L\left(\frac{\partial n_{e}}{\partial T}-\frac{\partial n_{o}}{\partial T}\right)
$$

From material parameters values available in [14], [15], we compute the sensitivity of the frequency difference $\Delta v$ to temperature and to EO applied voltage of, respectively, $1.41 \mathrm{GHz} / \mathrm{K}$ at $25^{\circ} \mathrm{C}$ and $1.56 \mathrm{MHz} / \mathrm{V}$. Thus the crystal temperature allows a coarse tunability of the frequency difference to the targeted value of $9.192 \mathrm{GHz}$. Experimentally the sensitivity coefficients are $1.4 \mathrm{GHz} / \mathrm{K}$ (Fig. 2) and $1.3 \mathrm{MHz} / \mathrm{V}$, in good agreement with the theoretical values.

\section{LASER CHARACTERIZATION}

First of all, the laser is characterized without any intra-cavity element. The laser threshold is obtained at $0.25 \mathrm{~W}$ of incident pump power. The laser output power reaches $83 \mathrm{~mW}$ for the maximal pump power limited by thermal effects in the

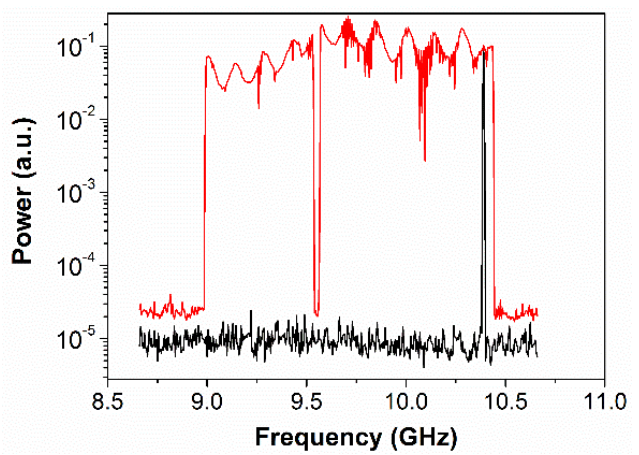

Fig. 2. RF spectrum of the beatnote of the two laser modes as measured on an $\mathrm{RF}$ spectrum analyzer. The black curve corresponds to the beatnote spectrum at a stabilized temperature. The red curve is the trace generated by the beatnote spectrum during a $1 \mathrm{~K}$ temperature sweep of the EO. Mode-hopes (around 9.5 and $10 \mathrm{GHz}$ ) are visible on this trace. semiconductor structure; the laser emission exhibits then a multimode spectrum centered at $855 \mathrm{~nm}$. With the $50 \mu \mathrm{m}$-thick Fabry-Perot etalon, the laser operates in a single mode emission with a wavelength tunability of almost $5 \mathrm{~nm}$. With all intracavity elements, the laser output power decreases to $26 \mathrm{~mW}$ at $852.1 \mathrm{~nm}$ corresponding to $13 \mathrm{~mW}$ per polarization. The laser threshold increases to $0.35 \mathrm{~W}$ due to the additional optical losses induced by the cavity elements (Fig 3). The fine tunability of the cross-polarized laser frequencies is achieved with the rotation of the Fabry-Perot etalon and the adjustment of the cavity length.

The optical spectrum of the dual-frequency laser around $852.1 \mathrm{~nm}$ is measured with a Fourier transform optical spectrum analyzer with a resolution of $2 \mathrm{pm}$ (Fig. 4). Polarizationresolved measurements show no presence of side modes, confirming the emission is single-mode on each polarization. The beatnote is evidenced by mixing the cross-polarized modes with a polarizer oriented at $45^{\circ}$ of their axes, and coupling the beam into a fiber-coupled fast GaAs photodiode. The freerunning beatnote spectrum around $9.2 \mathrm{GHz}$ has a spectral linewidth at $-3 \mathrm{~dB}$ below $600 \mathrm{kHz}$ on a time scale of $300 \mathrm{~ms}$, measured with a resolution bandwidth of $100 \mathrm{kHz}$ (Fig. 5). The beatnote linewidth is limited by residual uncorrelated mechanical and thermal fluctuations.

\section{LASER STABILIZATION}

Under free-running conditions, the stability of the two laser

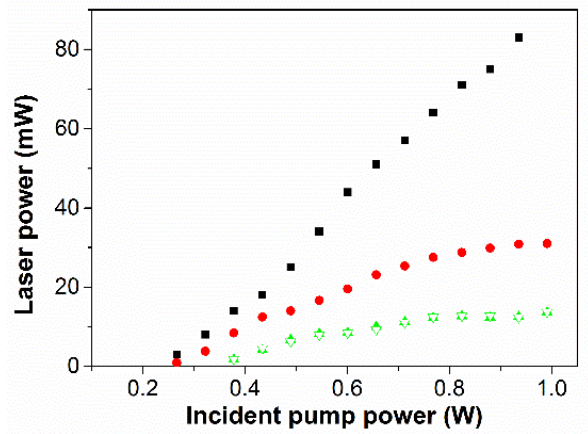

Fig. 3. Laser power characterization for different cavity configurations. Black: without any intracavity elements. Red: with all intracavity elements, for single frequency emission at $852.1 \mathrm{~nm}$. Green: with all intracavity elements, for bifrequency emission at $852.1 \mathrm{~nm}$ and for each polarization.

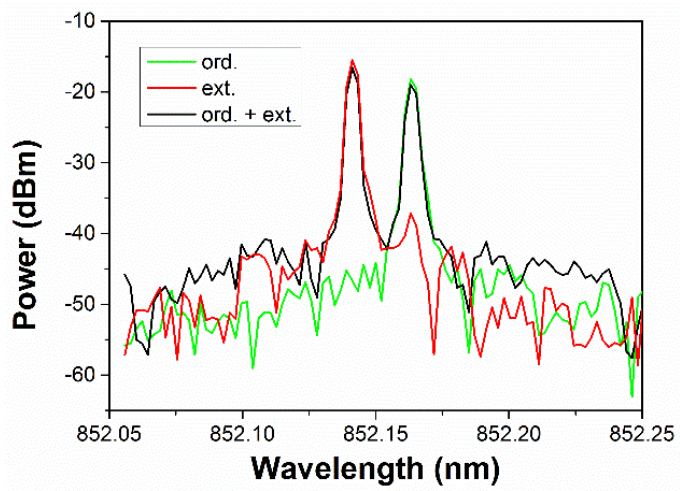

Fig. 4. Optical spectrum of the dual frequency laser measured with a resolution of $2 \mathrm{pm}$ for each polarization and with both polarizations. 


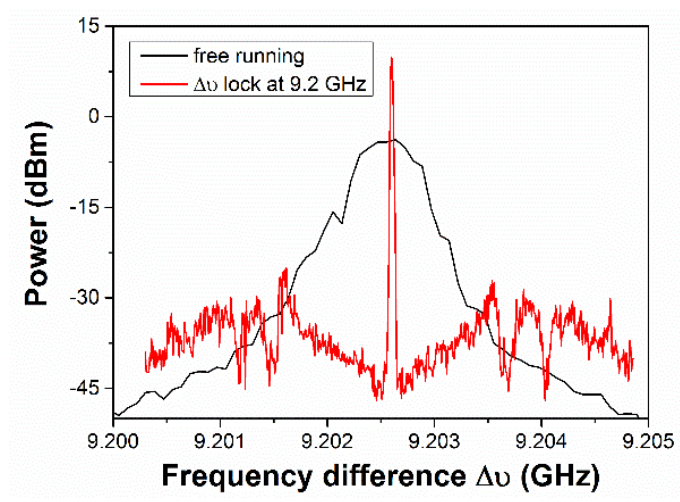

Fig. 5. Beatnote frequency spectrum in free-running operation (RBW $=100$ $\mathrm{kHz}$ ) and in locked operation (RBW $=10 \mathrm{kHz}$ ), at $9.2 \mathrm{GHz}$. The spectral linewidth of the beatnote in locked operation is limited by the spectrum analyzer resolution.

lines is not compatible with the interrogation of Cs atoms in a CPT-based atomic clock, which requires a stable emission at the Cs atomic transition and a high-purity optically-carried microwave signal. The laser emission has thus to be further stabilized. In this section we detail the stabilization of the ordinary-polarized laser line onto a hyperfine Cs atomic transition, and the concurrent lock of the frequency difference $\Delta v$ onto a RF oscillator.

At the output of the laser, the two cross-polarized modes are separated by a polarization beam splitter, and one $-30 \mathrm{~dB}$ optical isolator per polarization is used to prevent spurious optical feedback (Fig. 6). A fraction $(0.5 \mathrm{~mW})$ of the ordinarypolarized beam is injected into a saturated absorption set-up for its stabilization on a Cs atomic transition at $852.1 \mathrm{~nm}$. Finally, both beams are recombined with the same polarization axis in order to measure the beatnote frequency and lock it on the RF frequency reference.

\section{A. Optical frequency stabilization at Cs transition}

The laser frequency of the ordinary polarized beam is locked

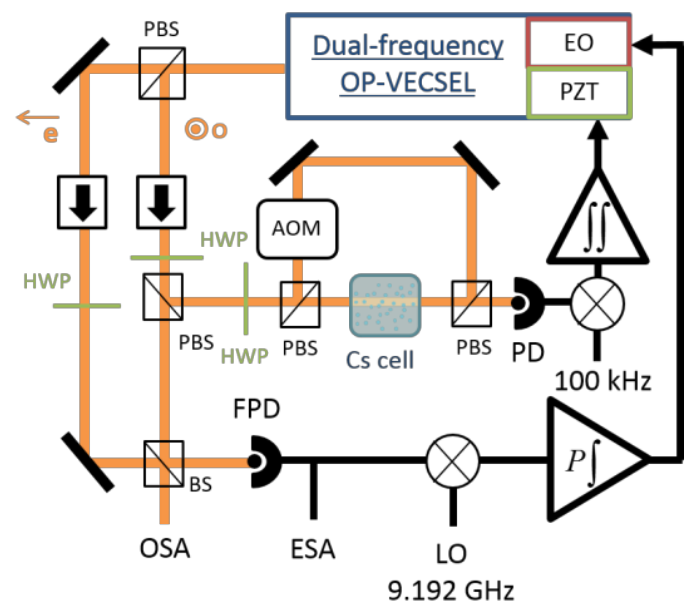

Fig. 6. Stabilization set-up. EO: electro-optic crystal, PZT: piezo-electric transducer, BS: beam splitter, PBS: polarization beam splitter, HWP: halfwave plate, AOM: acousto-optic modulator, PD: photodiode, FPD: fast photodiode, LO: local oscillator, PJ: proportional integrator corrector, $\iint$ : double-integrator corrector, OSA: optical spectrum analyzer, ESA: electrical spectrum analyzer. onto a Doppler-free transition ( $F=4$ towards $\left.F^{\prime}=4^{\prime} / 5^{\prime}\right)$ of the Cs $\mathrm{D}_{2}$ line using a pump-probe saturated absorption technique in a Cs cell at room temperature [16], as shown in Fig. 6. An acousto-optic modulator is used to modulate the counterpropagating pump beam at $100 \mathrm{kHz}$. An error signal $\epsilon_{1}$ is generated by heterodyne detection of the probe beam transmitted through the Cs cell. This method avoids the direct modulation of the pump diode current, which would simultaneously introduce a modulation of the laser power. The correction is made by a low-frequency two-integration stage servo loop on the piezo-electric transducer glued to the output coupler. We measure a static sensitivity coefficient of the optical frequency to the error signal of $12 \mathrm{MHz} / \mathrm{V}$.

\section{B. Frequency difference stabilization}

The frequency difference $\Delta v$ between the two laser modes is locked, using an optical-phase lock loop [17], onto a commercial RF synthesizer (Agilent E8257D) used as a local oscillator (LO), which generates a stable RF reference at the frequency $v_{L O}$. The two laser frequencies are mixed on a fast GaAs photodiode (Fig 6). The subsequent beatnote signal is amplified and mixed with the RF reference to generate an error signal proportional to $\left(\Delta v-v_{L O}\right)$. The error signal is then amplified by a high-bandwidth proportional-integrator corrector and applied to the intracavity electro-optic crystal to compensate for the frequency-difference fluctuations.

Fig. 5 compares the beatnote spectra of the free-running laser and of the laser when the frequency difference $\Delta v$ is locked to the RF reference at $9.2 \mathrm{GHz}$. The RF spectrum is significantly improved within a $1 \mathrm{MHz}$ band. This band, corresponding to the servo loop bandwidth, results from the open loop gain and is limited by the servo-loop response delay, most likely induced by electronics.

The signal-to-noise ratio, measured between the peak maximum and its minimum close to the frequency carrier, is 95 $\mathrm{dB} / \mathrm{Hz}$. The two servo-loops - of the absolute frequency difference on a $\mathrm{Cs}$ atomic transition and of the frequency difference on the RF local oscillator - operate together for 30 minutes under laboratory conditions. Better working conditions with acoustic isolation of the set-up are being implemented to achieve a longer operating time for the stabilized laser.

\section{LASER NOISE AND DYNAMICS}

The noise properties of the stabilized laser emission are investigated in order to evaluate the contribution of the dualfrequency OP-VECSEL to the performance of a CPT-Ramsey atomic clock. We study three main noise sources: the laser intensity noise, the laser frequency noise and the beatnote phase noise.

\section{A. Laser intensity noise}

Polarization-resolved measurements show no significant difference in the relative intensity noise (RIN) levels between the two modes on a $[10 \mathrm{~Hz} ; 100 \mathrm{kHz}]$ frequency range. A white noise floor of $-115 \mathrm{~dB} / \mathrm{Hz}$ for each polarization has been measured directly at the laser output (Fig. 7). Moreover, we observe experimentally that the two frequency-stabilization 


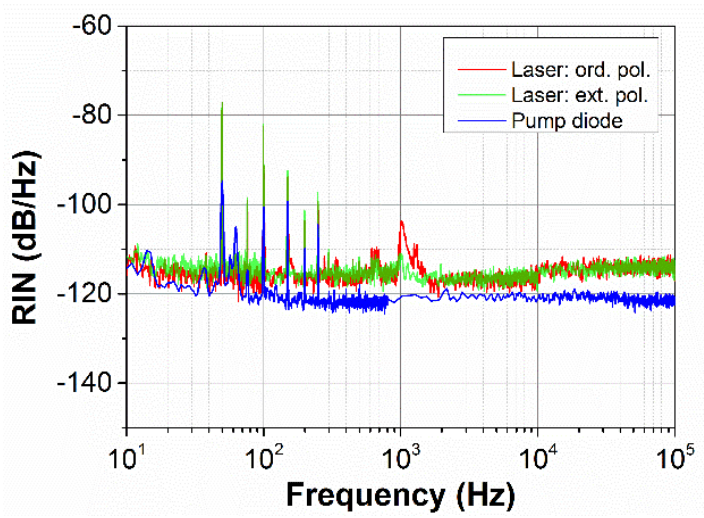

Fig. 7. Spectral density of the laser relative intensity noise for each polarization, and of the pump relative intensity noise.

servo-loops do not disturb the RIN levels. The laser intensity noise is actually limited by the pump intensity noise, whose level is $-120 \mathrm{~dB} / \mathrm{Hz}$ at frequencies below $100 \mathrm{kHz}$. We observe a RIN conversion factor from the pump to the laser of $+5 \mathrm{~dB}$, compatible with our numerical estimations following [18], within the bandwidth of the pump-to-laser RIN transfer estimated at $90 \mathrm{MHz}$ under our laser operating conditions. Furthermore, we have verified that the RIN of the pump diode is not limited by its injected current noise, and we suspect the mode partition noise resulting from the competition between the longitudinal modes to be the main limiting factor of the pump RIN [19]. A lower RIN of our dual-frequency laser should thus be achievable by using a single-mode pump source. High power tapered laser diodes at $670 \mathrm{~nm}$ with quasi-single mode emission have been demonstrated [20], and are considered as good candidates.

\section{B. Laser frequency noise}

The laser frequency noise reveals the fluctuations of the absolute optical frequency as compared to the Cs transition. To quantify the power spectral density of the laser frequency noise $S_{v_{o p t}}(f)$, we measured the power spectral density of the error signal $\epsilon_{1}$ with a Fast Fourier Transform spectrum analyzer, and normalized it by the static sensitivity coefficient of the set-up (12 MHz/V). We believe that, for $f \leq 1 \mathrm{kHz}$, the major contribution to $S_{v_{\text {opt }}}(f)$ comes from mechanical fluctuations in the cavity. Indeed, the contribution of the pump-induced

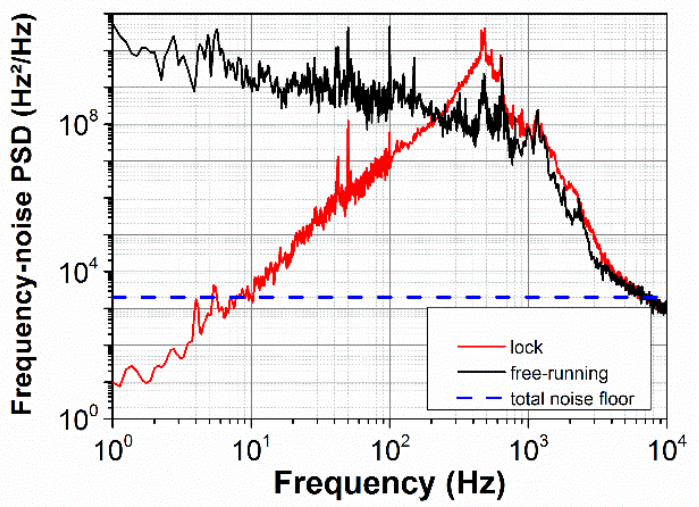

Fig. 8. Power spectrum density of the frequency noise of the ordinary polarized mode, under free-running operation (black line) and under locked operation (red line). Total noise floor originating from the RIN conversion into the servo loop is represented in the dashed line. thermal fluctuations inside the semiconductor chip - converted into a frequency noise through changes of the optical cavity length - is estimated to $6 \times 10^{5} \mathrm{~Hz}^{2} / \mathrm{Hz}$ in the bandwidth of the thermal effects [21], a few orders of magnitude below the observed frequency noise under free-running operation (Fig. 8). In locked operation, the low-frequency mechanical and thermal noise is suppressed on a bandwidth of $600 \mathrm{~Hz}$, limited by the piezo-transducer resonance. At $1 \mathrm{~Hz}$, we measure a frequency noise below the total noise floor, which means that a $60 \mathrm{~dB}$ noise reduction has been achieved. In fact, since this measurement is realized within the servo-loop, it does not reveal the noise issued from the loop components themselves. We believe that the actual noise floor comes from the conversion of the laser $R I N$ into frequency noise through the servo-loop, and is estimated at $2.0 \times 10^{3} \mathrm{~Hz}^{2} / \mathrm{Hz}$.

\section{Beatnote phase noise}

The local oscillator (LO) which provides the RF interrogation signal is a key element in an atomic frequency reference. More specifically in CPT atomic clocks, the LO signal is optically carried by the two coherent laser lines. Conversion from electrical to optical $(\mathrm{E} / \mathrm{O})$ domain is obtained by locking the laser frequency difference $\Delta v$ to the LO frequency $v_{L O}$. In a perfect situation, the spectral purity of the LO, i.e. its phase noise, is reproduced on the optically-carried RF signal. However the $\mathrm{E} / \mathrm{O}$ conversion is a source of additional noises, originating from the optical phase-lock loop and the laser itself. To characterize the power spectrum density (PSD) of the beatnote additive phase noise (or residual phase noise) $S_{\Phi_{\text {Add }}}(f)$ originating from the OPLL and the laser, we use the set-up presented in Fig. 9 which rejects the LO noise as a common noise source for both signals measured by FPD1 et FDP2. A tunable delay line (or phase-shifter) ensures that the LO signal and the beatnote signal measured with FPD2 are inphase. Fig. 10 shows $S_{\Phi_{A d d}}(f)$ under free-running and locked operations (for a frequency difference $\Delta v$ locked at the LO frequency of $9.6 \mathrm{GHz}$ ). A level of $-105 \mathrm{dBrad}^{2} / \mathrm{Hz}$ on the 100 $\mathrm{Hz}-5 \mathrm{kHz}$ frequency range is measured, below the LO typical phase noise. This result is comparable with measurements obtained on solid-state dual-frequency lasers [6]. At frequencies above $5 \mathrm{kHz}$, the noise level increases up to -90 $\mathrm{dBrad}^{2} / \mathrm{Hz}$ at $1 \mathrm{MHz}$, which corresponds to the optical phaselock loop bandwidth. This excess noise is due to the reduction of the OPLL corrector gain at high frequency and thus could be

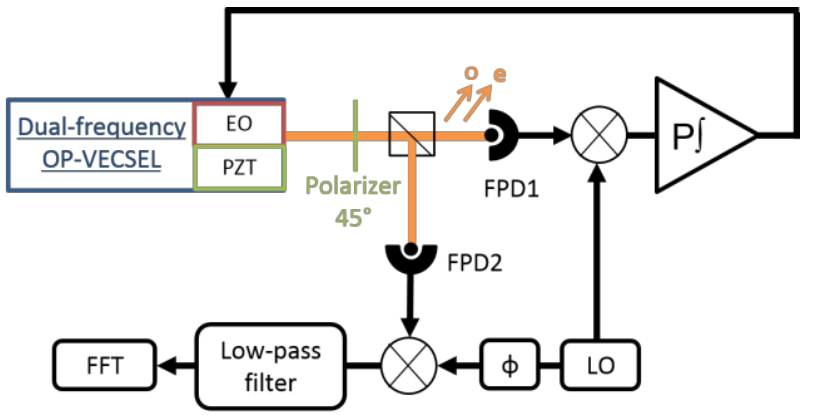

Fig. 9. Additive phase noise measurement set-up, including the frequency difference lock loop with the proportional-integrator corrector. FPD1 and FPD2: fast photodiodes, LO: Local oscillator, $\Phi$ : phase-shifter, $\mathrm{P}$ : proportional integrator corrector, FFT: Fast Fourier Transform spectrum analyzer. 


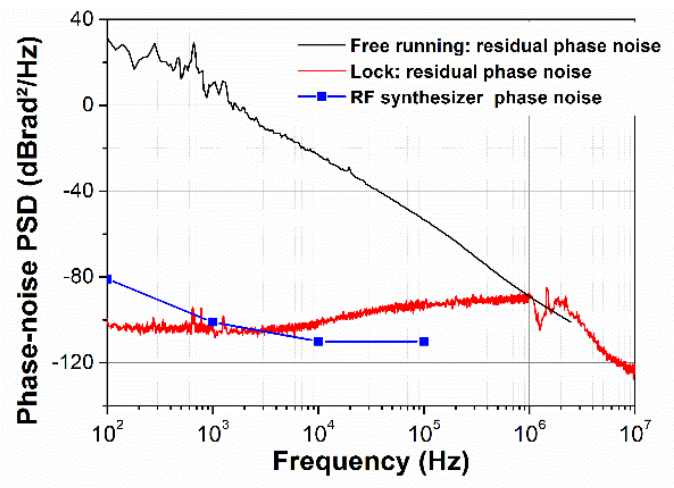

Fig. 10. Power spectrum density of the beatnote phase noise due to the laser and the optical phase-lock loop contribution (residual phase noise) under freerunning operation and under locked operation (LO frequency: 9.6 GHz) compared to the standard phase noise of the LO used.

better compensated. For frequencies above $1 \mathrm{MHz}$, a $1 / f^{2}$ slope is observed which is a typical behaviour of such lasers under free-running conditions [22].

\section{EVALUATION OF THE CLOCK FREQUENCY STABILITY}

In this section, we evaluate the laser noise contribution to the atomic clock performance and therefore the ultimate clock frequency stability, which would be reached with a CPT atomic clock based on our dual-frequency laser source. We will consider the CPT atomic clock using temporal Ramsey-like pulsed interrogation developed at Observatoire de Paris SYRTE [23].

The atomic clock scheme is described in Fig. 11. The two cross-polarized laser modes illuminate a cell filled with Cs atoms and a buffer gas. Before the cell, an acousto-optic modulator is used for the temporal pulse shaping of the atoms interrogation. The clock sequence of duration $T_{c}=6 \mathrm{~ms}$ is composed of a first pumping pulse $\tau_{p}=2 \mathrm{~ms}$, a free-evolution time $T_{R}=4 \mathrm{~ms}$ and finally a very short detection pulse $\tau_{d}=$ $25 \mu \mathrm{s}$, during which the power transmitted by the Cs cell is measured.

The clock stability is characterized by the relative frequency fluctuations of the LO oscillator, around the atomic ground state splitting. It is calculated by the Allan standard deviation $\sigma_{y}(\tau)$, equal to the quadratic sum of three contributions resulting from the laser intensity noise (IN), the laser frequency noise (FN) and the beatnote phase noise amplified by the Dick effect [24].

$$
\sigma_{y}^{2}(\tau)=\sigma_{I N}^{2}+\sigma_{F N}^{2}+\sigma_{D i c k}^{2}
$$

The major contribution of the laser power fluctuations (IN) is on the amplitude noise of the detected signal $S(t)$. The atomic

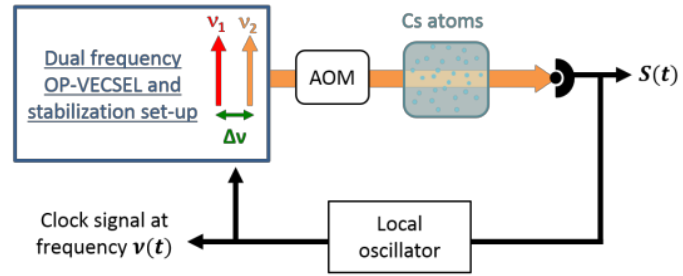

Fig. 11. General scheme for a CPT-Ramsey atomic clock using Cs atoms trapped in a cell.
TABLE I

CALCULATIONS OF ALLAN STANDARD DEVIATION FOR EACH LASER NOISE CONTRIBUTION

\begin{tabular}{lll}
\hline \hline Laser noise contribution & Max noise level & \multicolumn{1}{c}{$\begin{array}{c}\text { Allan standard } \\
\text { deviation } \sigma_{y}(\tau)\end{array}$} \\
\hline Intensity noise $(R I N)$ & $-115 \mathrm{~dB} / \mathrm{Hz}$ & $1.5 \times 10^{-12} \tau^{-1 / 2}$ \\
Frequency noise & $10^{10} \mathrm{~Hz}^{2} / \mathrm{Hz}$ & $7.7 \times 10^{-14} \tau^{-1 / 2}$ \\
Beatnote phase noise & $-90 \mathrm{dBrad}^{2} / \mathrm{Hz}$ & $2.7 \times 10^{-13} \tau^{-1 / 2}$
\end{tabular}

clock signal $S(t)$ is sensitive to this contribution on a bandwidth of $1 / \tau_{d}=40 \mathrm{kHz}$. On the contrary, the laser optical frequency noise $S_{v_{\text {opt }}}(f)$ impacts the clock frequency stability during the pumping time $\tau_{p}$. Indeed, the laser frequency noise is converted into an amplitude noise of the clock signal on a bandwidth of $700 \mathrm{~Hz}$, through the fluctuations of the density of atoms trapped in the dark state. Finally, the Dick effect arises from the lack of information on the optically-carried RF frequency during the pumping of the $\mathrm{Cs}$ atoms. Therefore, the pumping time corresponds to a dead time during which frequency fluctuations of the RF frequency can not be corrected [25].The Dick effect bandwidth includes all frequencies above the clock interrogation frequency $1 / T_{c}$. As discussed in V.B, the optically-carried RF frequency noise (or beatnote frequency noise) originates from the LO intrinsic frequency noise $S_{v_{O L}}(f)$, the OPLL frequency noise $S_{v_{O P L L}}(f)$, and the laser frequency noise $S_{v_{L}}(f)$. The beatnote frequency noise can be computed from the beatnote additive phase noise $S_{\Phi_{\text {Add }}}(f)=S_{v_{\text {Add }}}(f) /$ $f^{2}$ evaluated previously.

Each laser contribution to the Allan standard deviation is estimated following the approach described in [20] (Table 1). For the sake of simplicity, we consider white noise spectral densities at their measured maximum level for the three contributions. The commercial RF synthesizer used as LO would also contribute to $5 \times 10^{-13} \tau^{-1 / 2}$ to the clock stability. From these calculations, we estimate a clock stability, limited mainly by the laser intensity noise, of $\sigma_{y}(\tau)=1.6 \times 10^{-12} \cdot \tau^{-1 / 2}$. Though this value is higher than the state-of-the-art CPTRamsey atomic clock [26], it is not a fundamental limit of the atomic clock. In order to reach better clock performance, we should adapt the clock set-up. For example, an increase of the detection time from $25 \mu \mathrm{s}$ to $125 \mu \mathrm{s}$ could reduce the $R I N$ contribution to Allan standard deviation below $9 \times 10^{-13} \cdot \tau^{-1 / 2}$. It would also decrease the influence of the Dick effect, but may reduce the CPT signal $S(t)$ as well. The cause and consequences of such a compromise are detailed in [4], [23]. The clock sensitivity to the laser RIN might also be reduced by monitoring the laser beam power in front of the atomic cell and normalizing the CPT signal. With such a normalization, a threefold reduction of the contribution of the laser RIN to the Allan standard deviation has been demonstrated for an initial RIN floor level of $-115 \mathrm{~dB} / \mathrm{Hz}$ [24]. Alternatively we may add a power stabilization loop of the pump laser or use a single-mode tapered laser diode as a pump source (cf V.B). Eventually, once we change the current LO for an ultra-low noise microwave chain [4] and reduce the laser intensity noise contribution below the Dick limit, the clock frequency stability should be below $3 \times 10^{-13} \cdot \tau^{-1 / 2}$ corresponding to the current state-of-theart of such atomic clocks [4]. 


\section{CONCLUSION}

We have demonstrated the dual-frequency / dual-polarization emission of an OP-VECSEL at $852 \mathrm{~nm}$, with a frequency difference of $9.2 \mathrm{GHz}$. It is based on the introduction of a controlled birefringence within the laser cavity, which results in two cross-polarized lasers lines. A compact prototype has been designed, which allows a fine tunability of the laser optical frequencies. The output power reaches $13 \mathrm{~mW}$ per line. The laser dual-frequency emission is stabilized by means of two servo-loops, on a Cs atomic transition in a saturated-absorption setup and on a stable RF oscillator. Under this working operation, the laser noise properties have been carefully investigated. Measurements show a maximum noise level of $115 \mathrm{~dB} / \mathrm{Hz}$ for the laser relative intensity noise and -90 $\mathrm{dBrad}^{2} / \mathrm{Hz}$ for the additive phase noise.

Finally, we have theoretically evaluated the impact of the residual noise of our dual-frequency laser source on the shortterm frequency stability of a CPT-Ramsey atomic clock. We demonstrate that a clock stability of $1.6 \times 10^{-12} \cdot \tau^{-1 / 2}$ should be achieved, and would be limited by the laser intensity noise contribution. The latter might be reduced by changing either the laser or the clock set-up; the impact of these changes will have to be investigated through an exhaustive study of the total signal-to-noise ratio of the CPT signal. Eventually we expect that the laser contribution to clock frequency stability would be limited by the Dick effect below $3 \times 10^{-13} \tau^{-1 / 2}$, paving the way for future compact atomic clocks with $1 \times 10^{-13} \tau^{-1 / 2}$ frequency stability level.

\section{REFERENCES}

[1] J. Vanier, "Atomic clocks based on coherent population trapping: a review," Appl. Phys. B, vol. 81, no. 4, pp. 421-442, Jul. 2005.

[2] S. Knappe, V. Shah, P. D. D. Schwindt, L. Hollberg, J. Kitching, L.A. Liew, and J. Moreland, "A microfabricated atomic clock," Appl. Phys. Lett., vol. 85, no. 9, pp. 1460-1462, 2004.

[3] T. Zanon, S. Guerandel, E. de Clercq, D. Holleville, N. Dimarcq, and A. Clairon, "High contrast Ramsey fringes with coherentpopulation-trapping pulses in a double lambda atomic system.," Phys. Rev.Lett., vol. 94, no. 19, p. 193002, May 2005.

[4] J. Danet, M. Lours, S. Guérandel, and E. de Clercq, "Dick effect in a pulsed atomic clock using coherent population trapping," to be published in IEEE Ultrason. Ferroelectr. Freq. Control, 2014.

[5] X. Liu, J.-M. Mérolla, S. Guérandel, C. Gorecki, E. de Clercq, and R. Boudot, "Coherent-population-trapping resonances in buffer-gasfilled Cs-vapor cells with push-pull optical pumping," Phys. Rev. A, vol. 87, no. 1, p. 013416, Jan. 2013.

[6] G. Pillet, L. Morvan, M. Brunel, F. Bretenaker, D. Dolfi, M. Vallet, J. P. Huignard, A. Le Floch, and S. Member, "Dual-Frequency Laser at $1.5 \mu \mathrm{m}$ for Optical Distribution and Generation of HighPurity Microwave Signals," J. Light.Technol., vol. 26, no. 15, pp. 2764-2773, 2008.

[7] R. Czarny, M. Alouini, C. Larat, M. Krakowski, and D. Dolfi, "THz-dual-frequency $\mathrm{Yb} 3+: \mathrm{KGd}(\mathrm{WO} 4) 2$ laser for continuous wave THz generation through photomixing," Electron. Lett., vol. 40, no. 15, pp. 2-4, 2004.

[8] G. Baili, M. Alouini, D. Dolfi, F. Bretenaker, I. Sagnes, and A. Garnache, "Shot-noise-limited operation of a monomode highcavity-finesse semiconductor laser for microwave photonics applications.," Opt. Lett., vol. 32, no. 6, pp. 650-652, Mar. 2007.

[9] G. Baili, L. Morvan, M. Alouini, D. Dolfi, F. Bretenaker, I. Sagnes, and A. Garnache, "Experimental demonstration of a tunable dualfrequency semiconductor laser free of relaxation oscillations.," Opt. Lett., vol. 34, no. 21, pp. 3421-3, Nov. 2009.
[10] F. A. Camargo, J. Barrientos, G. Baili, L. Morvan, D. Dolfi, D. Holleville, S. Guerandel, I. Sagnes, P. Georges, and G. Lucas-leclin, "Coherent Dual-Frequency Emission of a Vertical External-Cavity Semiconductor Laser at the Cesium D2 Line," IEEE Photonics Technol. Lett., vol. 24, no. 14, pp. 1218-1220, 2012.

[11] O. G. Okhotnikov, Semiconductor Disk Lasers: Physics and Technology. 2010.

[12] B. Cocquelin, D. Holleville, G. Lucas-Leclin, I. Sagnes, a. Garnache, M. Myara, and P. Georges, "Tunable single-frequency operation of a diode-pumped vertical external-cavity laser at the cesium D2 line," Appl. Phys. B, vol. 95, no. 2, pp. 315-321, Jan. 2009.

[13] V. Pal, P. Trofimoff, B.-X. Miranda, G. Baili, M. Alouini, L. Morvan, D. Dolfi, F. Goldfarb, I. Sagnes, R. Ghosh, and F. Bretenaker, "Measurement of the coupling constant in a twofrequency VECSEL.," Opt. Express, vol. 18, no. 5, pp. 5008-5014, Mar. 2010.

[14] N. Ter-Gabrielyan, V. Fromzel, and M. Dubinskii, "Linear thermal expansion and thermo-optic coefficients of YVO4 crystals the 80$320 \mathrm{~K}$ temperature range," Opt. Mater. Express, vol. 2, no. 11, pp. 1624-1631, Oct. 2012.

[15] I. Dolev, A. Ganany-Padowicz, O. Gayer, A. Arie, J. Mangin, and G. Gadret, "Linear and nonlinear optical properties of MgO:LiTaO3," Appl. Phys. B., vol. 96, no. 2-3, pp. 423-432, Apr. 2009.

[16] W. Demtröder, Atoms, Molecules and Photons: an introduction to atomic-, molecular and quantum physics. Berlin/Heidelberg: Springer-Verlag, 2006, pp. 444-446.

[17] J. Yao, "A Tutorial on Microwave Photonics," IEEE Photonics Soc. Newsl., pp. 4-12, 2012.

[18] G. Baili, F. Bretenaker, M. Alouini, L. L. Morvan, D. Dolfi, and I. Sagnes, "Experimental Investigation and Analytical Modeling of Excess Intensity Noise in Semiconductor Class-A Lasers," J. Light. Technol., vol. 26, no. 8, pp. 952-961, 2008.

[19] M. Yamada, "Theory of mode competition noise in semiconductor lasers," Quantum Electron. IEEE J., vol. QE-22, pp. 1052-1059, 1986.

[20] B. Sumpf, P. Adamiec, M. Zorn, H. Wenzel, and G. Erbert, "Nearly diffraction-limited tapered lasers at $675 \mathrm{~nm}$ with $1-\mathrm{W}$ output power and conversion efficiencies above 30\%," IEEE Photonics Technol. Lett., vol. 23, no. 4, pp. 266-268, Feb. 2011.

[21] A. Laurain, M. Myara, G. Beaudoin, I. Sagnes, and A. Garnache, "Multiwatt-power highly-coherent compact single-frequency tunable vertical-external-cavity-surface-emitting-semiconductorlaser.," Opt. Express, vol. 18, no. 14, pp. 14627-36, Jul. 2010.

[22] S. De, A. El Amili, I. Fsaifes, G. Pillet, G. Baili, F. Goldfarb, M. Alouini, I. Sagnes, and F. Bretenaker, "Phase Noise of the Radio Frequency (RF) Beatnote Generated by a Dual-Frequency VECSEL," J. Light. Technol., vol. 32, no. 7, pp. 1307-1316, Apr. 2014.

[23] J. Danet, M. Lours, P. Yun, S. Guérandel, and E. de Clercq, "Frequency Instability Investigations on a Cs Cell Clock Based on Pulsed Coherent Population Trapping," in European Frequency and Time Forum, 2013, pp. 586-589.

[24] J. Danet, O. Koslova, P. Yun, S. Guérandel, and E. de Clereq, "Compact atomic clock prototype based on coherent population," to be published in Eur. Phys. Journal, Web Conf., 2014.

[25] G. J. Dick, "Local oscillator induced instabilities in trapped ion frequency standards," in Proc. 19th Precise Time and Time Interval (PPTI) Applications and Planning Meeting, 1987, pp. 133-147.

[26] R. Boudot, S. Guerandel, E. de Clercq, N. Dimarcq, and A. Clairon, "Current Status of a Pulsed CPT Cs Cell Clock," IEEE Trans. Instrum. Meas., vol. 58, no. 4, pp. 1217-1222, Apr. 2009. 